\title{
CARL FRIED, ARZT UND DICHTER
}

REINALDO BOSSMANN

Am 2. Juni dieses Jahres starb in São Paulo ein grosser Eurpaeer: Professor Dr. Carl Fried, Arzt von internationalem Ruf, Gelehrter und Dichter. Seine Wiege stand in der alten deutschen Bischofs - und Kaiserstadt Bamberg. Der spaetere Dichter gruesst die Stadt seiner Kindheit, nach Jahren muehsamer und beschwerlicher Erdenwanderung, mit den heimatlichen Versen:

Stadt der sieben sanften Huegel, sonnentrunkenen, heiligen Roms frankenlaendisch heiterer Spiegel;

Stadt des altersgrauen Doms mit den trotzig zarten Tuermen, die in ihrer Ruhestatt

Bischoefe und Kaiser schirmen:

Alte, treue, deutsche Stadt.

Stadt am stillen gruenen Fluss, dem die himmelhohen Eichen auf jahrtausendaltem Fuss tief herab die $Z$ weige reichen. Stadt der dunklen Foehrenwaelder: und der gruenen Wintersaat. Stadt der dunklen Foehrenwaelder: schoene, reiche, deutsche Stadt. 
Nach dem Besuch des humanistischen Gymnasiums, das inm die Humaniora vermittelte und seine Anlagen zum Humanisten und zur Humanitaet foerderte, war der junge Fried fuer das Studium der Medizin an den Universitaeten Muenchen, Kiel und Berlin bestens vorbereitet. Der Freiwillige erlebte den 1. Weltkrieg als Frontarzt auf den verschiedensten Kriegsschauplaetzen. Die vaterlaendische und aerztliche Pflicht riefen ihn zur Teilnahme an den Kaempfen imBaltikum in die Eiserne Division. Dafuer hat inn dann das Regime unter Hitler als "Dank" in das Konzentrationslager Buchenwald verwiesen. Fried hat jedoch davon nie gesprochen; denn seiner Natur lag jegliche Aeusserung ueber Gewalttaten fern, auch in der dichterischen Aussage. Nach dem Frontdienst wurde er Assistent bei Professor Doederlein in Muenchen, spaeter wirkte er als Oberarzt und Mitarbeiter bei Professor Heidenhain in Worms. Mit Heidenhain gab er 1922 das grundlegende Werk "Die Roentgenbestrahlung der bakteriellen Entzuendlungen" heraus. Auch das 1928 erschienene Werk von Prof. Heidenhain "Uber das Problem der boesartigen Geschwuelste" nennt Fried als verantwortlichen Mitarbeiter fuer den experimentellen Teil der Untersuchung. Ueber 10 Jahre leitete dann Dr. Fried als Chefarzt ein grosses Institut fuer Strahlenheilkunde in der schlesischen Haupstadt Breslau. Seine Zwangsemigration aus Deutschland erfolgte 1939. In den Vereinigten Staaten fand er als Dozent der Universitaet New York voruebergehende Aufnahme. Doch bald wurde er zur Gruendung und wissenschaftlichen Leitung des Roetgen- und Radium-Instituts São Francisco de Assis nach São Paulo berufen. Als Universitaetslehrer half er hier mit, die angehenden Aerzte auszibilden. Etwa 150 von ihm veroeffentlichte wissenschaftliche Arbeiten berichten von seinen reichen Erfahrungen und Forschungsergebnissen auf dem Gebiet der Strahlenheilkunde. Dr. Fried war Mitglied der Deutschen Roentgengesellschaft und korrespondierendes Mitglied mehrerer wissenschaftlicher Gesellschaften in aller Welt. Als Wissenschaftler ist er aber immer der Arzt fuer seine zahlreichen Patienten geblieben, die bei ihm Heilung und Linderung ihrer Leiden suchten. 
Seinen Kollegen war er stets ein verlaesslicher Helfer und Berater im Fuer und Wider der Vor- Nachteile von Strahlenbehandlungen in medizinisch besonders gelagerten Faellen.

Wenn das Tagewerk des Arztes Fried getan war, widmete er sich seiner Familie, seinem grossen Freundeskreis und der Dichtkunst. Im Gedichte "Einfaches Leben" heisst es in der Schlussstrophe:

Doch wenn der Abendschatten faellt, geht er ins kleine Haus zurueck, nicht ohne einen milden Blick vom Tor noch in die gute Welt.

Die gute Welt waren sein Idealistentum, sein Humanismus, sein Glaube an das Gute im Menschen, verbunden mit einer unerschuetterlichen Liebe fuer alles Edle und Milde. Als Dichter bekennt er sich zu den Versen:

Grad' auf dem Worte "lieb" war jetzt ein Tritt:

der Stiefelabsatz hatte schoen getroffen!

Vergebens, hier auf guten Sinn zu hoffen:

wo Liebe stirbt, stirbt Menschlichkeit meist mit.

Als Dichter leidet der Arzt - aehnlich wie bei Carossa - mit seinnen Kranken und empfindet mit ihnen den menschlichen Schmerz:

Und mancher Kranke, dem ich Schweres kuende, sieht auch die Blumen, und er ahnt vielleicht, dass aus dem Zimmer das Gefuehl nicht weicht und dass ich - als Mensch mit ihm empfinde.

Die Sehnsucht nach seiner deutschen Heimat war Dr. Frieds staendiger Begleiter. Doch ist sie ihm in der Schwere nicht Schicksal geworden. Seine erasmische Natur, sein mildes Verstehen, sein feiner, lebensnaher, nie verletzender $\mathrm{Hu}$ mor liessen ihn immer den guetigen Ausgleich finden. InGedichten gab er dieser Sehnsucht Ausdruck: 
Wo du daheim warst, ist nicht mehr dein Heim.

Und wo dein Heim ist, bist du nicht daheim.

Gingst du auch "heim", es waer nicht mehr daheim.

Wo du auch gehst, du findest nimmer heim.

Es war ihm noch vergoennt, bevor ihn ein boeses Leiden auf das Krankenlager zwang, mit seiner geliebten deutschen Heimat Wiedersehen zu feiern. Von der Erfuellung dieses seines Herzenswunsches kuenden tiefempfundene Verse, in Schmerz und Glueck.

Schon als junger Frontarzt schrieb Dr. Fried seine Lyrik. Den "Kriegsgedichten" folgten spaeter die "Arztgedichte" und die "Gedichte eines Auswanderers". Seine "Brasilienlyrik" spricht fuer den feinen Sinn, der Wahlheimat auch nur das Schoene, Milde, Edle und Versoehnende abzulauschen. Diese Verse - eine Auswahl hat B. A. Aust, São Paulo, im Jahre 1954 herausgegeben - stellen ihn in die Reihe der "Deutschen Dichter in Brasilien".

Carl Fried:

\section{DIE IPES}

Ipês, die prangend goldenen Glocken laeuten den Fruehling ein, wie jedes Jahr.

Wie es im Leben immer war, der Tod ist nah fuer die, die sich vergeuden.

Ein heisser Wind, ein schwueler Sommertag: die Glocken fallen bleich und fahl, der Fruehlingsbaum steht schwarz und kahl der Trauer harrend, die nun kommen mag.

Jedoch der Fruehling hoert nicht auf zu strahlen: die Azaleen leuchten rot; vergessen ist der Opfertod mit dem Ipês ihr goldenes Leuchten zahlen. 


\section{BRASILIANISCHER HERBST}

Ist dies wahrhaftig Herbst, wenn milde Sonne die bunten Fliesen vor den Hausern waermt und in des Feigenbaumes hoher Krone, wenns kuehlt, das Vogelheer noch zirpt und laermt?

Ist dies ein Herbst, wenn in jung-gruenen Gaerten zum zweitenmal erbluehn die Azaleen und beerenrot die Erdbeerstauden werden, als muesse alles Reifen jetzt geschehn?

Ich kannte einst den Herbst mit kalten Stuermen, trueb, lichtlos und mit toedlich fruehen Frost. Die Wetterfahnen kreischten auf den Tuermen, man fror, - kam Waerme nicht vom Ofenrost.

Muss man denn froesteln, weil es Winter vird? Alterndes Herz, in Sorgen eingesponnen, lern von Brasilien Herbst, dass der sich irrt, der glaubt, nichts koenne seinen Herbst besonnen. 